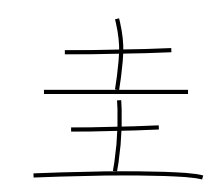

\title{
Burnside kei
}

by

\section{Maciej Niebrzydowski and Józef H. Przytycki (Washington, DC)}

\begin{abstract}
This paper is motivated by a general question: for which values of $k$ and $n$ is the universal Burnside kei $\bar{Q}(k, n)$ finite? It is known (starting from the work of M. Takasaki (1942)) that $\bar{Q}(2, n)$ is isomorphic to the dihedral quandle $Z_{n}$ and $\bar{Q}(3,3)$ is isomorphic to $Z_{3} \oplus Z_{3}$. In this paper, we give a description of the algebraic structure for Burnside keis $\bar{Q}(4,3)$ and $\bar{Q}(3,4)$. We also investigate some properties of arbitrary quandles satisfying the universal Burnside relation $a=\cdots a * b * \cdots * a * b$. Invariants of links related to the Burnside kei $\bar{Q}(k, n)$ are invariant under $n$-moves.
\end{abstract}

1. Introduction. A $k e i$, 主, also called an involutory quandle, was introduced by Mituhisa Takasaki in 1942 [Tak] as an abstract algebra $(Q, *)$ with a binary operation $*: Q \times Q \rightarrow Q$ satisfying the following conditions:

(i) $a * a=a$ for any $a \in Q$,

(ii) $(a * b) * b=a$,

(iii) $(a * b) * c=(a * c) *(b * c)$ (right distributivity).

We use a standard convention for products in nonassociative algebras, called the left-normed convention, that is, whenever parentheses are omitted in a product of elements $a_{1}, a_{2}, \ldots, a_{n}$ of $Q$ then

$$
a_{1} * a_{2} * \cdots * a_{n}=\left(\left(\cdots\left(\left(a_{1} * a_{2}\right) * a_{3}\right) * \cdots\right) * a_{n-1}\right) * a_{n}
$$

(left association), for example, $a * b * c=(a * b) * c$. The conditions given in the above definition are related to Reidemeister moves (see Figure 1).

We will consider free keis with the universal relation

$$
r_{n}: \quad a=\cdots a * b * \cdots * a * b,
$$

2000 Mathematics Subject Classification: Primary 57M25; Secondary 55N99, 20 D99.

Key words and phrases: kei, quandle, involutory quandle, Burnside kei, Burnside group, $n$-move, quasigroup, Nakanishi Conjecture. 

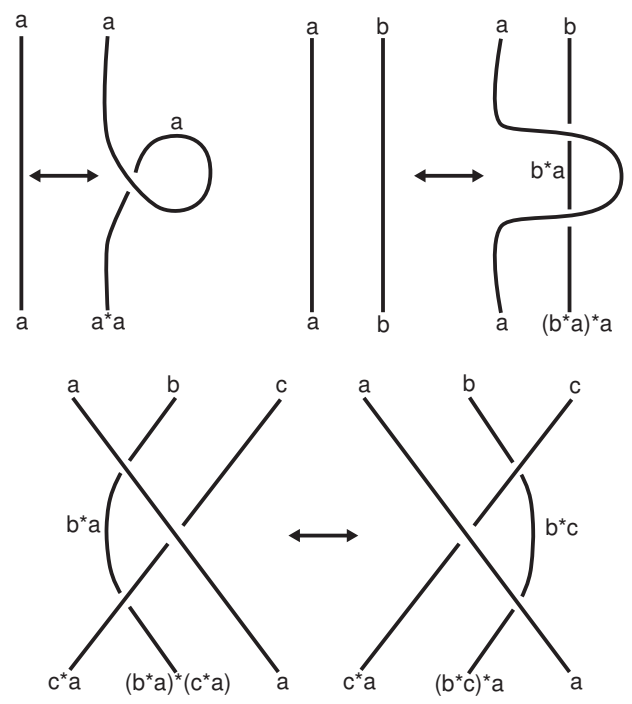

Fig. 1. Reidemeister moves and kei axioms
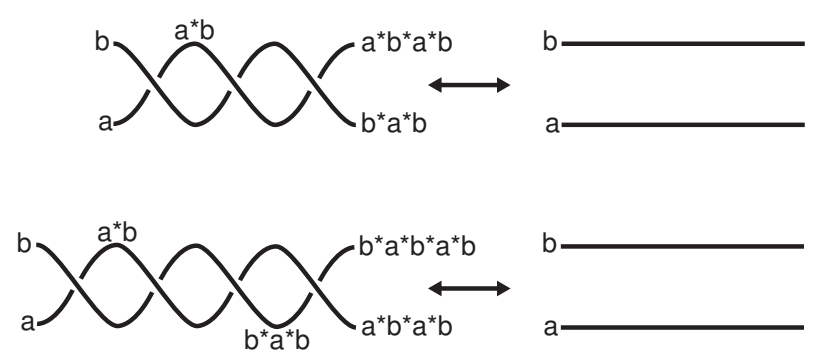

Fig. 2. Correspondence between $n$-moves and relation $r_{n}$

in which there are $n$ letters on the right hand side and $a, b$ are any elements of the quandle. We denote such a kei with $k$ generators by $\bar{Q}(k, n)$ and call it the universal Burnside kei.

In [Joy], D. Joyce associated an involutory quandle to a link. In a similar way we can associate to every link $L$ its $n$th Burnside kei, $\bar{Q}_{n}(L)$, by assigning generators to arcs of a fixed diagram of $L$, writing the relation of the form $u * v=w$ for each crossing (here $u$ and $w$ are generators corresponding to the under-arcs and $v$ is assigned to the over-arc) and adding the universal relation $r_{n}$.

The relation $r_{n}$ corresponds to local changes in a link diagram called $n$-moves. It follows that $\bar{Q}_{n}(L)$ is invariant under Reidemeister moves and $n$-moves. For example, $r_{3}: a=b * a * b$ corresponds to invariance under 3-moves and $r_{4}: a=a * b * a * b$ makes $\bar{Q}_{4}(L)$ invariant under 4-moves. 
Figure 2 illustrates this correspondence in the case $n=3,4$. In fact, $\bar{Q}_{n}(L)$ is also invariant under rational $n / m$-moves [D-I-P].

We notice that the relation $r_{3}: a=b * a * b$ is equivalent to $a * b=b * a$, in other words, $\bar{Q}(k, 3)$ is a free commutative kei on $k$ generators $\left({ }^{1}\right)$.

Problem 1. For which values of $k$ and $n$ is $\bar{Q}(k, n)$ finite? How many elements does it have?

In this paper, we focus on finitely generated commutative keis and keis satisfying the 4th universal Burnside relation $a=a * b * a * b$.

\section{Commutative keis}

2.1. ExAMPLES. Let us first recall that there are two well known classes of examples of finite commutative keis:

(1) dihedral kei, $Z_{3}$ (with $i * j=2 j-i=-j-i$ modulo 3 ), corresponding to Fox 3 -colorings, and its direct sums $Z_{3}^{n}$ with coordinatewise operation;

(2) the 3rd Burnside group, $B(k, 3)=\left\{x_{1}, \ldots, x_{k} \mid w^{3}=1\right.$ for any word $w\}$, with the core operation $a * b=b a^{-1} b$, and its quotients.

Notice that $B(k, 3)$ is a commutative kei as the equality $a * b=b * a$ follows from the identity $b a^{-1} b=a b^{-1} a$, which, in $B(k, 3)$, is equivalent to $\left(b a^{-1}\right)^{3}=1$. Our motivation for Problem 1 is a theorem by Burnside [Bu] stating that $B(k, 3)$ is a finite group.

2.2. Some properties of commutative keis. First, let us describe some general properties of involutory quandles satisfying the relation $r_{3}$. Any quandle is distributive from the right, but in the case of commutative keis, we also have distributivity from the left:

$$
c *(a * b)=(a * b) * c=(a * c) *(b * c)=(c * a) *(c * b) .
$$

From axiom (ii) in the definition of kei, it follows that for any $a, b \in Q$, there exists a unique $c \in Q$ such that $a=c * b$ (and obviously $c=a * b$ ).

Here, we mention that if we replace axiom (ii) with the above statement without the condition that $c=a * b$, we get a general definition of a quandle (see $[\mathrm{F}-\mathrm{R}]$ for a description of quandle theory from the historical perspective). For a survey on knot invariants derived from quandles see [Kam].

The equality $a=c * b$ is equivalent to

$$
a * c=b \quad \text { and } \quad c * a=b .
$$

$\left({ }^{1}\right)$ The commutativity relation should not be confused with the abelian condition, $(a * b) *(c * d)=(a * c) *(b * d)$, introduced in [Joy]. 
It follows that any commutative kei is a quasigroup $\left(^{2}\right)$ and the set $\{a, b, c=$ $a * b\}$ is a subquandle. If $m$ denotes the size of a finite commutative kei, then there are $\left(\begin{array}{c}m \\ 2\end{array}\right) / 3$ such 3 -element subquandles and each element $x \in Q$ belongs to $(m-1) / 2$ of them (choosing any element $p \in Q \backslash x$ determines the third element of the quandle, $x * p)$.

An involutory quandle $Q$ is said to be algebraically connected if for each pair $a, b$ in $Q$, there are $a_{1}, \ldots, a_{s} \in Q$ such that

$$
a * a_{1} * \cdots * a_{s}=b .
$$

We say that an involutory quandle is strongly algebraically connected if it is algebraically connected and $s=1$ in the above definition.

LEMMA 2. Any kei satisfying the universal relation $r_{k}$ for some odd $k$ is strongly algebraically connected.

Proof. Our relation $r_{k}$ now has the form

$$
a=b * a * b * \cdots * a * b .
$$

Using the first axiom of a quandle and the relation $x * y * z * y=x *(z * y)$, we can write $r_{k}$ as

$$
a=b * b * a * b * \cdots * a * b=\left\{\begin{array}{l}
b *(b * a * \cdots * a * b) \\
\text { or } \\
b *(a * b * \cdots * a * b)
\end{array}\right.
$$

depending on how many letters $a$ and $b$ we have in the relation $r_{k}$. In either case, in order to get from $a$ to $b$ we need to use only one operator (that can be written using $(k+1) / 2$ letters $a$ and $b)$.

Every algebraically connected quandle (not necessarily involutory) is a metric space if we define the distance between $x, y \in Q$ as the minimal number of operators needed to obtain one element from the other. A significant class of algebraically connected quandles is the knot quandles (see [Joy] for a definition). Since our metric is unchanged under isomorphism of quandles, some properties of the metric space (for example its diameter) are knot invariants. Lemma 2 states that the diameter of any quandle (that is, the diameter of the corresponding metric space) satisfying $r_{k}$ for some odd $k$ is 1 .

Two distinct elements $x$ and $y$ of a quandle $Q$ are called behaviorally equivalent if

$$
z * x=z * y \quad \text { for all } z \in Q
$$

$\left({ }^{2}\right)$ A quasigroup is a set $G$ together with a binary operation - with the property that for each $x, y \in G$, there are unique elements $w, z \in G$ such that $x \cdot w=y$ and $z \cdot x=y$. 
It turns out that there are no behaviorally equivalent elements in quandles of the sort considered above.

LEMMA 3. If $Q$ is a kei satisfying the relation $r_{k}$ for some odd $k$, then it has no behaviorally equivalent elements. Moreover, if $z * x=z * y$ for some $z \in Q$, then $x=y$.

Proof. Assume that $z * x=z * y$ for some $x, y, z \in Q$. In the case of a commutative kei we get the result immediately. Our assumption implies that $x * z=y * z$ and this forces the equality $x=y$. Let us consider the case $k>3$. Since the relation $r_{k}$ holds for all elements, we have

$$
z=x * z * x * \cdots * z * x \quad \text { (with } k \text { letters on the right), }
$$

which is equivalent to

$$
z * x * z=x * z * x * \cdots * z * x * z * x \quad \text { (with } k-2 \text { letters on the right) }
$$

and

$$
z=y * z * y * \cdots * z * y
$$

equivalent to

$$
z * y * z=y * z * y * \cdots * z * y * z * y .
$$

We can replace the initial assumption with

$$
z * x * z=z * y * z
$$

and use the relation $r_{k}$ to change this equation to:

$$
\begin{array}{r}
x * z * x * \cdots * z * x * z * x=y * z * y * \cdots * z * y * z * y \\
x * z * x * \cdots * z *(z * x)=y * z * y * \cdots * z * y * z * y \\
x * z * x * \cdots * z *(z * y)=y * z * y * \cdots * z * y * z * y \\
x * z * x * \cdots * z * y * z * y=y * z * y * \cdots * z * y * z * y .
\end{array}
$$

Now we can cancel the last four letters on both sides of the last equation. We repeat this reduction until we obtain $x=y$ or $x * z * x=y * z * y$. In the latter case, we use the first quandle axiom to write:

$$
\begin{gathered}
x * x * z * x=y * y * z * y \\
x *(z * x)=y *(z * y),
\end{gathered}
$$

and we reduce one more time to obtain $x=y$.

For every quandle $Q$, we can consider its operator group, $\operatorname{Op}(Q)$, generated by automorphisms $f_{x}: Q \rightarrow Q$ defined by $y f_{x}=y * x$. Behaviorally equivalent elements of $Q$ define equal elements in $\operatorname{Op}(Q)$. On the other hand, if there are no behaviorally equivalent elements in $Q$, then the map $x \mapsto f_{x}$ is injective and $Q$ is isomorphic to the union of the conjugacy classes of the images of the generators of $Q$ in $\operatorname{Op}(Q)$ (see also [Joy]). 
Corollary 4. Any kei $Q$ satisfying the universal relation $r_{k}$ for some odd $k$ embeds into the conjugation quandle of its operator group, $\operatorname{Conj}(\operatorname{Op}(Q))$ (with the quandle operation $f * g=g^{-1} f g, f, g \in \operatorname{Conj}(\mathrm{Op}(Q))$ )).

Absence of behaviorally equivalent elements enables us to prove the following theorem $\left(^{3}\right)$.

THEOREM 5. The order of a finite commutative kei $Q$ is a power of 3 .

Proof. Let $a, b \in Q$ and let $P$ be the 3-element subquandle $\{a, b, a * b\}$. From the right distributivity property, it follows that for any $x \in Q$, the set $S:=P * x=\{a * x, b * x,(a * b) * x\}$ is also a subquandle. We ask: what other elements of $Q$ send $P$ to $S$ ? There can be at most three such operators, sending $a$ to $a * x, b * x$ or $(a * b) * x$ (here we use the fact that two operators $x, y \in Q$ acting in the same way on one element are the same). Using Lemma 2 , we can find them easily:

(1) $a *(a * x)=\mathbf{x}$

(2) $a *(b * x)=b * x * a=\mathbf{x} * \mathbf{b} * \mathbf{a}$;

(3) $a *(a * b * x)=a * b * x * a=b * a * x * a=b *(x * a)=\mathbf{x} * \mathbf{a} * \mathbf{b}$.

From the left distributivity, it follows that the set of these three operators is a subquandle. We still need to check that operators (2) and (3) send $b$ and $a * b$ to $S$ :

$$
\begin{aligned}
b *(x * b * a) & =x * b * a * b=x *(a * b)=(a * b) * x ; \\
(a * b) *(x * b * a) & =b * a *(x * b * a)=b * a * a * b * x * b * a=b * x * b * a \\
& =x * b * b * a=a * x ; \\
b *(x * a * b) & =x * a * b * b=x * a=a * x ; \\
(a * b) *(x * a * b) & =a * b * b * a * x * a * b=a * x * a * b=x * a * a * b \\
& =x * b=b * x .
\end{aligned}
$$

In this way we obtain a partition of $Q$ into 3 -element disjoint subquandles of the form $\{x, x * b * a, x * a * b\}$, in which two elements belong to the same triple if they send $P$ to the same subquandle. This relation between elements is an equivalence relation but not a congruence $(u \sim v, s \sim t$ does not imply $u * s \sim v * t$ ), so we cannot simply form a quotient quandle. Instead, we define a natural quandle operation on triples:

$(x, x * b * a, x * a * b) \widehat{*}(y, y * b * a, y * a * b)=(x * y, x * y * b * a, x * y * a * b)$. The set of such triples, with the operation $\widehat{*}$, forms a commutative kei that is three times smaller than the original kei, $Q$. Thus we can use the inductive argument to conclude that the size of $Q$ is a power of 3 .

$\left({ }^{3}\right)$ After our paper appeared on the web, we received a very interesting e-mail from Michael Kinyon [Kin]. In particular, he brought to our attention the fact that finite commutative involutory quandles are exactly symmetric distributive quasigroups (or in combinatorial language, Hall triple systems) and such systems have order $3^{k}$ for some $k$. 
2.3. $\bar{Q}(4,3)$ has 81 elements. It was shown by M. Takasaki [Tak] that $\bar{Q}(2, n)$ is isomorphic to the dihedral quandle $Z_{n}$ and $\bar{Q}(3,3)$ is isomorphic to $Z_{3} \oplus Z_{3}$. Here we give a description of $\bar{Q}(4,3)$.

T. Ohtsuki wrote a computer program which helps to analyze the commutative kei. Using this program he found that $\bar{Q}(4,3)$ has 81 elements [Oht]. A different computation, involving the operator group of the quandle, was made by the first author $\left(^{4}\right)$. Here we follow, in a crucial point, Ohtsuki's approach to obtain a computer free proof.

THEOREM 6. $\bar{Q}(4,3)$ has 81 elements.

As noted by Takasaki, every element of the kei can be written in a leftnormed form (usually not uniquely). For example, in $\bar{Q}(4,3),(a * b) *(c * d)=$ $a * b * c * d * c=a * b * d * c * d=b * a * c * d * c=b * a * d * c * d=c * d * a * b * a=$ $c * d * b * a * b=d * c * a * b * a=d * c * b * a * b$. The length of a kei element $w$ associated to a particular kei presentation is the length of the shortest left-normed word representing $w$, in the chosen generators of kei.

LEMMA 7.

(i) Every element of $\bar{Q}(4,3)$, in the generating set $\{a, b, c, d\}$, is of length at most 7.

(ii) There are (at most) eight elements in $\bar{Q}(4,3)$ of length 7 and they have representatives:

$$
\begin{array}{ll}
a * b * c * d * b * c * d, & a * b * d * c * b * d * c, \\
b * a * c * d * a * c * d, & b * a * d * c * a * d * c, \\
c * a * b * d * a * b * d, & c * a * d * b * a * d * b, \\
d * a * b * c * a * b * c, & d * a * c * b * a * c * b .
\end{array}
$$

Proof. We use brackets [] to stress for which group of letters our properties are used. The brackets [ ] (unlike ( )) do not change the left-normed convention. Let $\left\{x_{0}, x_{1}, x_{2}, x_{3}\right\}=\{a, b, c, d\}$. We have the following identities in $\bar{Q}(4,3)$.

$$
x_{0} * x_{1} * x_{2} * x_{0}=x_{0} * x_{2} * x_{1} .
$$

Indeed, $x_{0} * x_{1} * x_{2} * x_{0}=x_{1} * x_{0} * x_{2} * x_{0}=x_{1} *\left(x_{0} * x_{2}\right)=x_{0} * x_{2} * x_{1}$.

$$
w * x_{0} * x_{1} * x_{0}=w * x_{1} * x_{0} * x_{1} .
$$

Indeed, $w * x_{0} * x_{1} * x_{0}=w *\left(x_{1} * x_{0}\right)=w *\left(x_{0} * x_{1}\right)=w * x_{1} * x_{0} * x_{1}$.

$$
\begin{aligned}
x_{0} * x_{1} * x_{2} * x_{3} * x_{2} & =\left(x_{0} * x_{1}\right) *\left(x_{2} * x_{3}\right)=\left(x_{2} * x_{3}\right) *\left(x_{0} * x_{1}\right) \\
& =x_{2} * x_{3} * x_{0} * x_{1} * x_{0} .
\end{aligned}
$$

$\left({ }^{4}\right)$ Michael Kinyon kindly informed us that the fact that $\bar{Q}(4,3)$ has 81 elements also follows from the result that the smallest nonassociative commutative Moufang loop is of order 81 (see [Bel]). 
(4) $x_{0} * x_{1} * x_{2} * x_{3} * x_{2} * x$ is reducible to a word of length 4 for $x=x_{i}$, $i=0,1,2,3$,

for example $x_{0} * x_{1} * x_{2} * x_{3} * x_{2} * x_{1}=\left(x_{0} * x_{1}\right) *\left(x_{2} * x_{3}\right) * x_{1}=x_{2} * x_{3} * x_{1} * x_{0}$.

$$
x_{0} * x_{1} * x_{2} * x_{3} * x_{0} * x_{1}=x_{0} * x_{1} * x_{3} * x_{2} * x_{0} .
$$

Indeed, $x_{0} * x_{1} * x_{2} * x_{3} * x_{0} * x_{1} * x_{0}=\left(\left(x_{0} * x_{1}\right) *\left(x_{2} * x_{3}\right)\right) * x_{2} *\left(x_{0} * x_{1}\right)=$ $\left(x_{2} * x_{3}\right) *\left(x_{0} * x_{1} * x_{2}\right)=\left(x_{0} * x_{1} * x_{2}\right) *\left(x_{2} * x_{3}\right)=x_{0} * x_{1} * x_{3} * x_{2}$.

$$
x_{0} * x_{1} * x_{2} * x_{3} * x_{0} * x_{3}=x_{0} * x_{2} * x_{1} * x_{3} * x_{0} .
$$

Indeed, $x_{0} * x_{1} * x_{2} * x_{3} * x_{0} * x_{3}=x_{1} * x_{0} * x_{2} * x_{3} * x_{0} * x_{3} \stackrel{(1)}{=} x_{1} * x_{0} * x_{2} *$ $x_{0} * x_{3} * x_{0} \stackrel{(2)}{=} x_{0} * x_{2} * x_{1} * x_{3} * x_{0}$.

$$
x_{0} * x_{1} * x_{2} * x_{3} * x_{1} * x_{2}=x_{3} * x_{2} * x_{1} * x_{0} * x_{2} * x_{1} .
$$

Indeed, $x_{0} * x_{1} * x_{2} * x_{3} * x_{1} * x_{2}=\left(\left(x_{0} * x_{1}\right) *\left(x_{2} * x_{3}\right)\right) *\left(x_{1} * x_{2}\right)=$ $\left(\left(x_{3} * x_{2}\right) *\left(x_{1} * x_{0}\right)\right) *\left(x_{2} * x_{1}\right)=x_{3} * x_{2} * x_{1} * x_{0} * x_{2} * x_{1}$.

$$
\begin{aligned}
x_{0} * x_{1} * x_{2} * x_{3} * x_{1} * x_{2} * x_{3} & =x_{0} * x_{2} * x_{3} * x_{1} * x_{2} * x_{3} * x_{1} \\
& =x_{0} * x_{3} * x_{1} * x_{2} * x_{3} * x_{1} * x_{2} .
\end{aligned}
$$

Indeed,

$$
\begin{aligned}
x_{0} * x_{1} * x_{2} * x_{3} * x_{1} *\left[x_{2} * x_{3} * x_{2}\right] & \stackrel{(2)}{=}\left(x_{0} * x_{1}\right) * x_{2} * x_{3} * x_{1} * x_{3} * x_{2} * x_{3} \\
& =\left(x_{1} * x_{0} * x_{2} * x_{3} * x_{1} * x_{3}\right) * x_{2} * x_{3} \\
& \stackrel{(6)}{=}\left(x_{1} * x_{2} * x_{0} * x_{3} * x_{1} * x_{2}\right) * x_{3} \\
& \stackrel{(5)}{=} x_{1} * x_{2} * x_{3} * x_{0} * x_{1} * x_{3} \\
& =x_{2} * x_{1} * x_{3} * x_{0} * x_{1} * x_{3} \\
& \stackrel{(7)}{=} x_{0} * x_{3} * x_{1} * x_{2} * x_{3} * x_{1}
\end{aligned}
$$

as required.

$$
x_{0} * x_{1} * x_{2} * x_{3} * x_{1} * x_{2} * x_{3} * x_{0}=x_{0} * x_{1} * x_{3} * x_{2} * x_{1} * x_{3} * x_{2} .
$$

This equality is the most difficult and allows us to complete the proof of Lemma 7. We follow Ohtsuki's analysis of his computer computation. He noticed that the key point is to use the commutation identity

$$
\begin{aligned}
\left(x_{0} * x_{1} * x_{2} * x_{3} * x_{1} * x_{2}\right. & \left.* x_{3} * x_{0}\right) * x_{2} \\
& =x_{2} *\left(x_{0} * x_{1} * x_{2} * x_{3} * x_{1} * x_{2} * x_{3} * x_{0}\right)
\end{aligned}
$$

and to show that the last expression can be reduced to $x_{0} * x_{1} * x_{3} * x_{2} * x_{1} * x_{3}$ by properties (1)-(8). This is done in Lemma 8 below.

LEMMA 8. $x_{2} *\left(x_{0} * x_{1} * x_{2} * x_{3} * x_{1} * x_{2} * x_{3} * x_{0}\right)=x_{0} * x_{1} * x_{3} * x_{2} * x_{1} * x_{3}$.

Proof. To improve readability, we omit $*$ in the presentation of words in this proof. Using the identity $w x y x=w(y x)=w(x y)=w y x y$ seven times 
we obtain

$$
x_{2}\left(x_{0} x_{1} x_{2} x_{3} x_{1} x_{2} x_{3} x_{0}\right)=x_{2} x_{0} x_{3} x_{2} x_{1} x_{3} x_{2} x_{1} x_{0} x_{1} x_{2} x_{3} x_{1} x_{2} x_{3} x_{0} .
$$

Now, after applying identities (1)-(8) several times, we obtain:

$$
\begin{aligned}
& \left(x_{2} x_{0} x_{3} x_{2}\right) x_{1} x_{3} x_{2} x_{1} x_{0} x_{1} x_{2} x_{3} x_{1} x_{2} x_{3} x_{0} \stackrel{(1)}{=} x_{2} x_{3} x_{0} x_{1} x_{3} x_{2} x_{1} x_{0} x_{1} x_{2} x_{3} x_{1} x_{2} x_{3} x_{0} \\
& =\left(x_{3} x_{2} x_{0} x_{1} x_{3} x_{2}\right) x_{1} x_{0} x_{1} x_{2} x_{3} x_{1} x_{2} x_{3} x_{0} \\
& \stackrel{(5)}{=} x_{3} x_{2} x_{1} x_{0} x_{3}\left[x_{1} x_{0} x_{1}\right] x_{2} x_{3} x_{1} x_{2} x_{3} x_{0} \stackrel{(2)}{=}\left(x_{3} x_{2} x_{1} x_{0} x_{3} x_{0}\right) x_{1} x_{0} x_{2} x_{3} x_{1} x_{2} x_{3} x_{0} \\
& \stackrel{(6)}{=}\left(x_{3} x_{1} x_{2} x_{0} x_{3} x_{1}\right) x_{0} x_{2} x_{3} x_{1} x_{2} x_{3} x_{0} \stackrel{(5)}{=}\left(x_{3} x_{1}\right) x_{0} x_{2} x_{3} x_{0} x_{2} x_{3} x_{1} x_{2} x_{3} x_{0} \\
& =\left(x_{1} x_{3} x_{0} x_{2} x_{3} x_{0}\right) x_{2} x_{3} x_{1} x_{2} x_{3} x_{0} \stackrel{(7)}{=} x_{2} x_{0} x_{3} x_{1} x_{0}\left[x_{3} x_{2} x_{3}\right] x_{1} x_{2} x_{3} x_{0} \\
& \stackrel{(2)}{=}\left(x_{2} x_{0}\right) x_{3} x_{1} x_{0} x_{2} x_{3} x_{2} x_{1} x_{2} x_{3} x_{0}=\left(x_{0} x_{2} x_{3} x_{1} x_{0} x_{2}\right) x_{3} x_{2} x_{1} x_{2} x_{3} x_{0} \\
& \stackrel{(5)}{=}\left(x_{2} x_{0}\right) x_{1} x_{3} x_{0} x_{3} x_{2} x_{1} x_{2} x_{3} x_{0}=\left(x_{0} x_{2} x_{1} x_{3} x_{0} x_{3}\right) x_{2} x_{1} x_{2} x_{3} x_{0} \\
& \stackrel{(6)}{=} x_{0} x_{1} x_{2} x_{3} x_{0}\left[x_{2} x_{1} x_{2}\right] x_{3} x_{0} \stackrel{(2)}{=}\left(x_{0} x_{1} x_{2} x_{3} x_{0} x_{1}\right) x_{2} x_{1} x_{3} x_{0} \\
& \stackrel{(5)}{=}\left(x_{0} x_{1} x_{3} x_{2} x_{0} x_{2}\right) x_{1} x_{3} x_{0} \stackrel{(6)}{=}\left(x_{0} x_{3}\right) x_{1} x_{2} x_{0} x_{1} x_{3} x_{0}=\left(x_{3} x_{0} x_{1} x_{2} x_{0} x_{1}\right) x_{3} x_{0} \\
& \stackrel{(7)}{=} x_{2} x_{1} x_{0} x_{3} x_{1}\left[x_{0} x_{3} x_{0}\right] \stackrel{(2)}{=}\left(x_{2} x_{1}\right) x_{0} x_{3} x_{1} x_{3} x_{0} x_{3}=\left(x_{1} x_{2} x_{0} x_{3} x_{1} x_{3}\right) x_{0} x_{3} \\
& \stackrel{(6)}{=}\left(x_{1} x_{0} x_{2} x_{3} x_{1} x_{0}\right) x_{3} \stackrel{(5)}{=}\left(x_{1} x_{0}\right) x_{3} x_{2} x_{1} x_{3}=x_{0} x_{1} x_{3} x_{2} x_{1} x_{3}
\end{aligned}
$$

as required.

We proved, in Lemma 7 , that $\bar{Q}(4,3)$ is finite, but in fact we can easily build, using Lemmas 7 and 8 and their proofs, the multiplication table of $\bar{Q}(4,3)$ with 81 elements. We still need to argue that the order of $\bar{Q}(4,3)$ is not smaller than 81 . One of the possible arguments is to show that application of the relations present in $\bar{Q}(4,3)$ does not lead to any further reductions in the number of elements of $\bar{Q}(4,3)$. This argument is quite laborious and mechanical as it requires a lot of similar computations, so it is suitable for a computer verification. A more sophisticated argument uses the kei epimorphism $p: \bar{Q}(4,3) \rightarrow Z_{3}^{3}$. The epimorphism $p$ is defined on generators of $\bar{Q}(4,3)$ as follows:

$$
p(a)=(0,0,0), \quad p(b)=(1,0,0), \quad p(c)=(0,1,0), \quad p(d)=(0,0,1) .
$$

For example, we have $p(a * b)=(2,0,0), p(a * c)=(0,2,0), p(a * d)=(0,0,2)$, $p(b * c)=(2,2,0), p(b * d)=(2,0,2), p(c * d)=(0,2,2)$.

From Theorem 5, it follows that it is enough to prove that $p$ is not a monomorphism. We notice that 


$$
p((a * b) *(c * d))=(1,1,1)=p((a * c) *(b * d))=p((a * d) *(b * d)) .
$$

However, the elements $(a * b) *(c * d)$ and $(a * c) *(b * d)$ are distinct in $\bar{Q}(4,3)$. To show that, we define a new kei homomorphism $q: \bar{Q}(4,3) \rightarrow B(4,3)$ which extends the identity map between the sets of generators. We have to check whether $q((a * b) *(c * d))=q((a * c) *(b * d))$. We have

$$
q((a * b) *(c * d))=q(c * d)(q(a * b))^{-1} q(c * d)=d c^{-1} d\left(b^{-1} a b^{-1}\right) d c^{-1} d,
$$

and similarly

$$
q((a * c) *(b * d))=d b^{-1} d\left(c^{-1} a c^{-1}\right) d b^{-1} d .
$$

Thus, we need to show that

$q((a * b) *(c * d))(q((a * c) *(b * d)))^{-1}=d c^{-1} d\left(b^{-1} a b^{-1}\right) d c^{-1} b d^{-1} c a^{-1} c d^{-1} b d^{-1}$

is not equal to 1 in $B(4,3)$. We reduce this problem, after conjugating by $d^{-1}$, to the question whether

$$
c^{-1} d b^{-1} a b^{-1} d c^{-1} b d^{-1} c a^{-1} c d^{-1} b \neq 1
$$

in the Burnside group $B(4,3)$. The above relation was verified both by using the computer algebra system GAP [GAP4], and by calculations in the associated Lie algebra of $B(4,3)$ made by Mietek Dąbkowski [Dab] (see [VL] for background information on Burnside groups). Thus, we have shown that $p$ is not a monomorphism and $\bar{Q}(4,3)$ has exactly 81 elements.

REMARK (alternative approach). As mentioned before (see page 215), the operator group of the quandle $Q, \operatorname{Op}(Q)$, is generated by the images of elements of $Q$ under the map $x \mapsto f_{x}$. Because of the relation

$$
f_{x * y}=f_{y}^{-1} f_{x} f_{y}
$$

which holds in the operator group for any $x, y \in Q, \operatorname{Op}(Q)$ is generated by the images of the generators of $Q$. In particular if $Q$ is generated by $n$ elements, then so is $\operatorname{Op}(Q)$.

As previously noted (see Corollary 4 and the comment preceding it), $\bar{Q}(4,3)$ embeds into its operator group. To simplify notation, we use the same symbols for quandle elements and their images in the corresponding operator group. From the second kei axiom it follows that the squares of generators (and therefore also the squares of conjugates of generators) are equal to the identity in $\operatorname{Op}(\bar{Q}(4,3))$. The relation $x * y=y * x$, which is true for all elements of $\bar{Q}(4,3)$, yields the relation $y x y=x y x$ (or $x y x y x y=1)$ in $\operatorname{Op}(\bar{Q}(4,3))$, where $x$ and $y$ belong to conjugacy classes of the generators of $\bar{Q}(4,3)$. Therefore, $\bar{Q}(4,3)$ can be embedded into the (possibly bigger) group $G_{(a, b, c, d)}^{(3)}$ with the following presentation:

$$
\left\{a, b, c, d \mid a^{2}=b^{2}=c^{2}=d^{2}=1, \text { xyxyxy }=1\right\},
$$


where $x$ and $y$ are any conjugates of the generators $a, b, c, d$. Using GAP, we found a finite presentation for $G_{(a, b, c, d)}^{(3)}$ as follows. We add to the presentation

$$
\left\{a, b, c, d \mid a^{2}=b^{2}=c^{2}=d^{2}=1\right\}
$$

all relations of the form $\left(w^{-1} \alpha w v^{-1} \beta v\right)^{3}=1$, where $\alpha, \beta \in\{a, b, c, d\}$ and $w$, $v$ are all possible two-letter words on the letters $a, b, c, d$. The group with this presentation is finite, and we can check that all required relations between conjugates of generators are satisfied ( $x y x y x y=1$ for any conjugates of $a, b, c, d)$. Again, using GAP, we computed that the order of this group is $118098=2 \cdot 3^{10}$ and that the number of elements in the union of the conjugacy classes of $a, b, c$ and $d$ is 81 (which is also the order of $\bar{Q}(4,3)$ ). Elements of these conjugacy classes form a 4-generator commutative kei with conjugation as a quandle operation, therefore their number cannot exceed the order of the free kei $\bar{Q}(4,3)$.

2.4. $\bar{Q}(4,3)$ as an extension of $Z_{3}^{3}$ by $Z_{3}$. We show that $\bar{Q}(4,3)$ is isomorphic to the quandle $\left(Z_{3} \times Z_{3}^{3}\right.$, $\left.\hat{*}\right)$, with the operation $\hat{*}$ defined as follows:

$$
\left(a_{1}, x_{1}\right) \hat{*}\left(a_{2}, x_{2}\right)=\left(a_{1} * a_{2}+c\left(x_{1}, x_{2}\right), x_{1} * x_{2}\right) .
$$

In this definition, + denotes addition in $Z_{3}, *$ is the quandle operation described in Example 2.1(1), and $c\left(x_{1}, x_{2}\right): Z_{3}^{3} \times Z_{3}^{3} \rightarrow Z_{3}$ is a function which satisfies the following conditions (related to the definition of kei):

(i) $c(x, x)=0$;

(ii) $c(x * y, y)=c(x, y)$;

(iii) $c\left(x_{1} * x_{3}, x_{2} * x_{3}\right)-c\left(x_{1} * x_{2}, x_{3}\right)=-c\left(x_{1}, x_{2}\right)+c\left(x_{2}, x_{3}\right)+c\left(x_{1}, x_{3}\right)$;

(iv) $c(x, y)=c(y, x)$.

The first condition corresponds to the first kei axiom, $(a, x) \hat{*}(a, x)=(a, x)$. The second condition is a consequence of the second kei axiom, $((a, x) \hat{*}$ $(b, y)) \hat{*}(b, y)=(a, x)$.

Condition (iii) follows from the right distributivity:

$$
\left(\left(a_{1}, x_{1}\right) \hat{*}\left(a_{2}, x_{2}\right)\right) \hat{*}\left(a_{3}, x_{3}\right)=\left(\left(a_{1}, x_{1}\right) \hat{*}\left(a_{3}, x_{3}\right)\right) \hat{*}\left(\left(a_{2}, x_{2}\right) \hat{*}\left(a_{3}, x_{3}\right)\right)
$$

leads to the following, after first computing the left and right sides of the above equation:

$$
\begin{aligned}
L= & \left(a_{1} * a_{2}+c\left(x_{1}, x_{2}\right), x_{1} * x_{2}\right) \hat{*}\left(a_{3}, x_{3}\right) \\
= & \left(a_{1} * a_{2} * a_{3}-c\left(x_{1}, x_{2}\right)+c\left(x_{1} * x_{2}, x_{3}\right), x_{1} * x_{2} * x_{3}\right), \\
R= & \left(a_{1} * a_{3}+c\left(x_{1}, x_{3}\right), x_{1} * x_{3}\right) \hat{*}\left(a_{2} * a_{3}+c\left(x_{2}, x_{3}\right), x_{2} * x_{3}\right) \\
= & \left(\left(a_{1} * a_{3}\right) *\left(a_{2} * a_{3}\right)+2 c\left(x_{2}, x_{3}\right)-c\left(x_{1}, x_{3}\right)\right. \\
& \left.+c\left(x_{1} * x_{3}, x_{2} * x_{3}\right),\left(x_{1} * x_{3}\right) *\left(x_{2} * x_{3}\right)\right) .
\end{aligned}
$$

From this we get

$$
c\left(x_{1} * x_{3}, x_{2} * x_{3}\right)-c\left(x_{1} * x_{2}, x_{3}\right)=-c\left(x_{1}, x_{2}\right)-2 c\left(x_{2}, x_{3}\right)+c\left(x_{1}, x_{3}\right) .
$$

Taking into account that we work modulo 3 , we get condition (iii). 
The last condition follows from the fact that we work with a commutative kei.

Condition (iii) turns each such $c$ into a twisted 2-cocycle in the second quandle cohomology group of $Z_{3}^{3}$ with $Z_{3}$ coefficients. Twisted quandle (co)homology theory was introduced in [C-E-S]. The authors described there a general method of obtaining a new quandle from a given quandle $X$ and Alexander quandle $A$, using a twisted 2-cocycle $\phi$. Such constructions, including the one we are describing, are called Alexander extensions of $X$ by $(A, \phi)$.

An example of a function $c$ satisfying all of the above conditions, is presented below. In order to represent a cocycle $c$ as a matrix, let us order the elements of $Z_{3}^{3}$ as follows:

1. $(0,0,0) ; 2$. $(0,0,1) ; 3$. $(0,0,2) ; 4$. $(0,1,0) ; 5$. $(0,1,1) ; 6$. $(0,1,2) ; 7$. $(0,2,0)$;

8. $(0,2,1)$; $9 .(0,2,2) ; 10 .(1,0,0) ; 11 .(1,0,1) ; 12 .(1,0,2) ; 13 .(1,1,0) ; 14 .(1,1,1)$; 15. $(1,1,2)$; 16. $(1,2,0)$; 17. $(1,2,1) ; 18 .(1,2,2) ; 19 .(2,0,0) ; 20$. $(2,0,1) ; 21 .(2,0,2)$;

22. $(2,1,0) ; 23$. $(2,1,1) ; 24$. $(2,1,2) ; 25$. $(2,2,0) ; 26$. $(2,2,1) ; 27$. $(2,2,2)$.

The entry $m_{i j}$ of the matrix $M$ given below equals the value $c(i, j)$ of the cocycle $c$ on the $i$ th and $j$ th elements of $Z_{3}^{3}$.

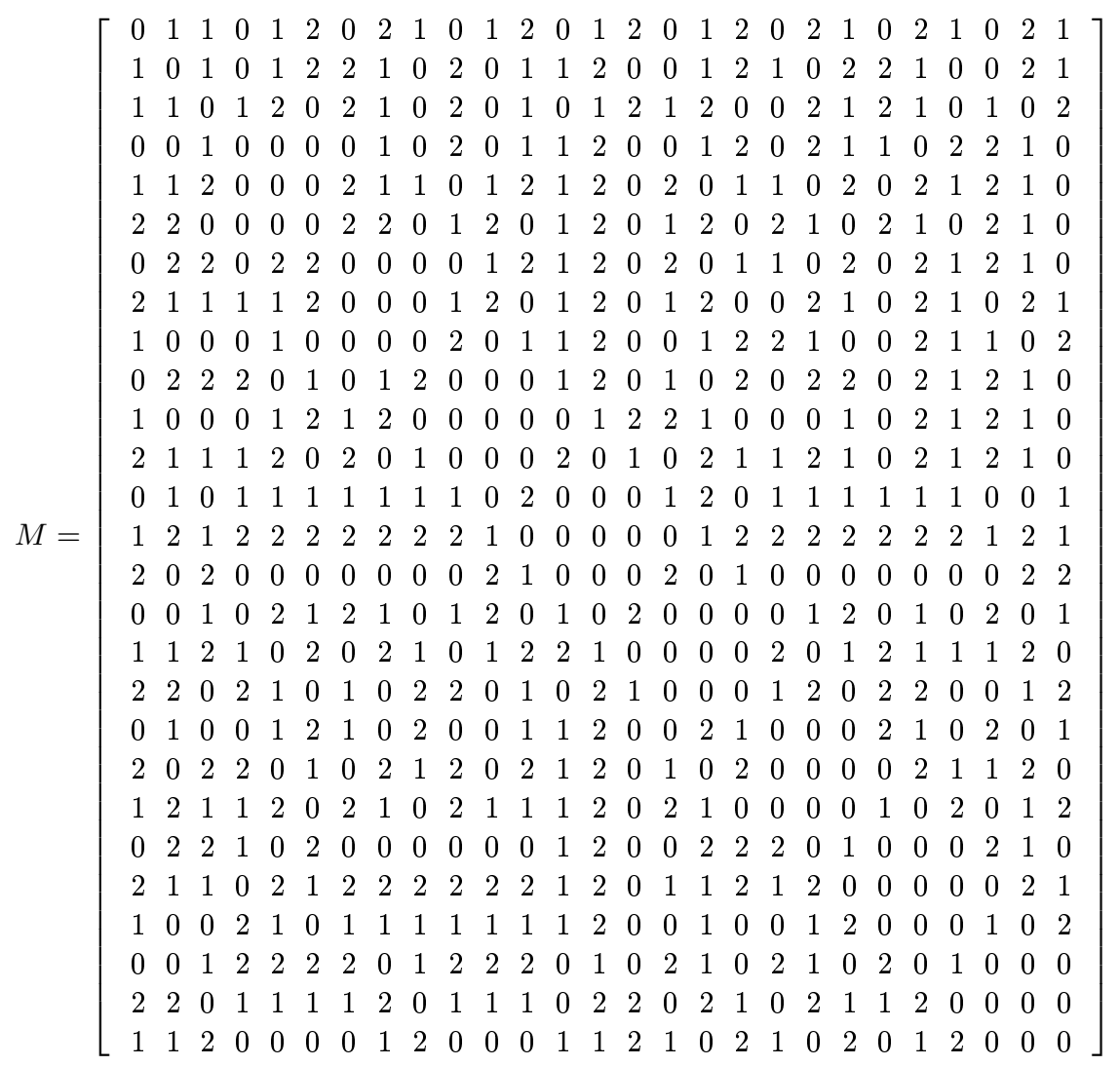


The existence of an isomorphism between $\bar{Q}(4,3)$ and $\left(Z_{3} \times Z_{3}^{3}\right.$, $\left.\hat{*}\right)$ follows from the following facts:

(i) $\left(Z_{3} \times Z_{3}^{3}\right.$, $)$ satisfies the kei axioms and relation $r_{3}$;

(ii) $\left(Z_{3} \times Z_{3}^{3}, \hat{*}\right)$ has 81 elements;

(iii) $\left(Z_{3} \times Z_{3}^{3}, \hat{*}\right)$ is generated by four elements: $(1,0,0,0),(0,1,0,0)$, $(0,0,1,0),(0,0,0,1)$ (for example it cannot be isomorphic to $\operatorname{Core}\left(Z_{3}^{4}\right)$, which has five generators as a kei).

3. $\bar{Q}(3,4)$ has 96 elements. The primary examples of keis satisfying the universal Burnside relation, $x=x * y * x * y$, are the dihedral kei $Z_{4}$, its direct sums, and the fourth Burnside groups and their quotients (with the core operation $x * y=y x^{-1} y$ ).

To get a lower bound on the order of $\bar{Q}(3,4)$, we can consider the group $G_{(a, b, c)}^{(4)}$ with presentation

$$
\left\{a, b, c \mid a^{2}=b^{2}=c^{2}=1,(x y)^{4}=1\right\},
$$

where $x$ and $y$ are any conjugates of the generators $a, b, c$. We obtain a finite presentation for $G_{(a, b, c)}^{(4)}$ in a similar way to the one described on page 221 . Using GAP we checked that this group has $8192=2^{13}$ elements and the size of the union of the conjugacy classes of the generators is 96 . The elements of these conjugacy classes form a 3-generator quandle (with conjugation as operation $*$ ) satisfying the relation $x=x * y * x * y$. Thus the order of the free kei $\bar{Q}(3,4)$ cannot be less than 96 . This time we cannot use Lemma 3 to obtain an upper bound on the size of $\bar{Q}(3,4)$ (see the remark after the proof of Theorem 6), so instead we will build a Cayley diagram for this quandle. This diagram has 96 vertices, therefore $\bar{Q}(3,4)$ has order 96. Below, we calculate some relations needed to build that diagram. Again, we use brackets [ ] to stress for which parts of words we use properties of $\bar{Q}(3,4)$. None of these properties can replace the first letter in the left-normed representatives of words in $\bar{Q}(3,4)$. For example a left-normed word starting with $a$ never equals a word starting with $b$. It follows that the diagram will consist of three disjoint parts that look the same when viewed as graphs (see Figure 3). Here we prove the most difficult relations in the Cayley graph, their numbers correspond to the numbers in Figure 3. The vertices of the Cayley graph represent the elements of $\bar{Q}(3,4)$. The solid arcs represent multiplication from the right by the generator $a$; two kinds of dashed arcs denote multiplication by respectively $b$ and $c$.

(1) We need to prove the relation

$$
a * b * c * a * b * a=a * b * c * a * b
$$




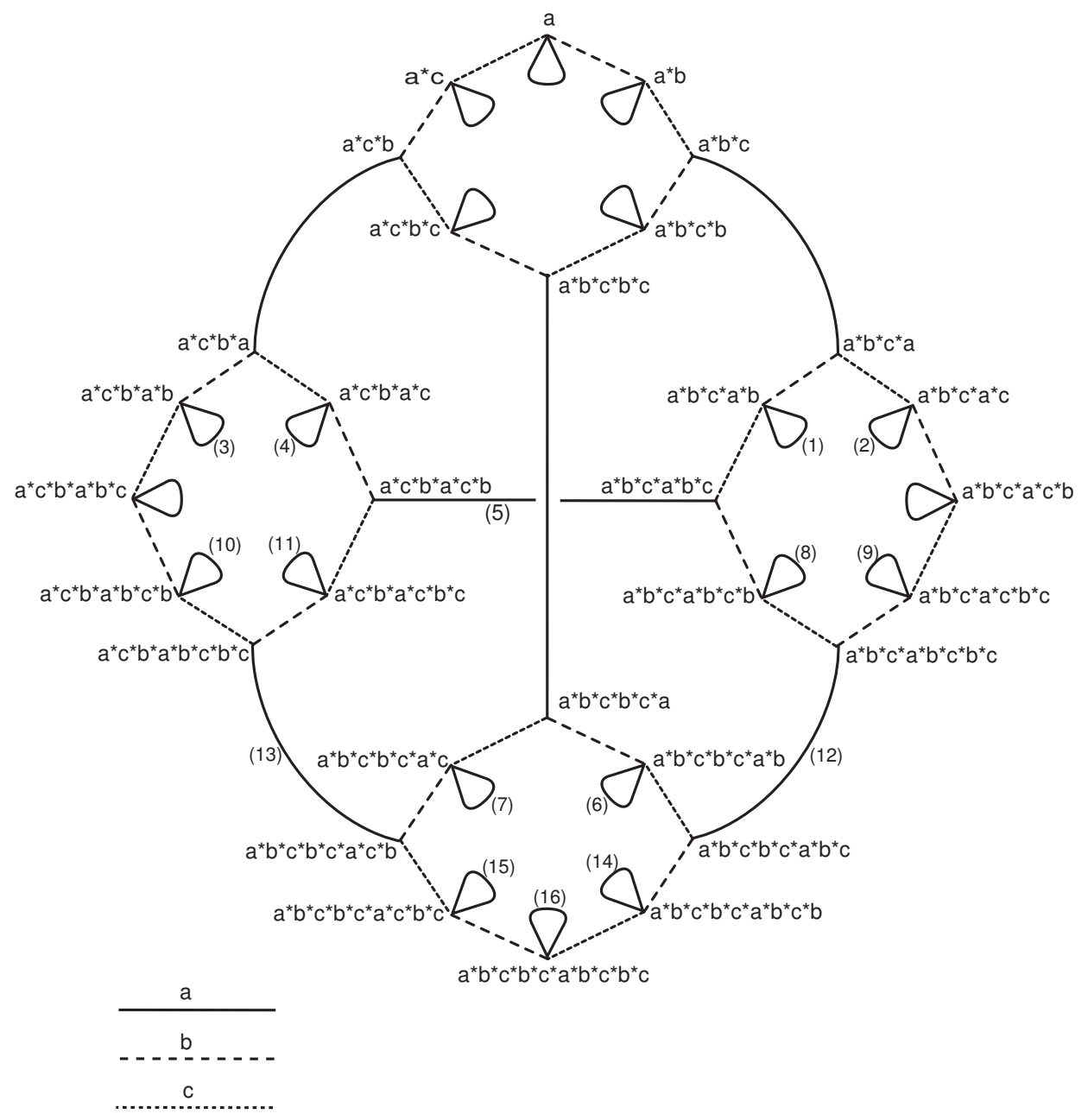

Fig. 3. A component of the Cayley diagram for $\bar{Q}(3,4)$

(corresponding to a loop at the vertex representing the element $a * b * c * a * b$ ):

$$
\begin{aligned}
{[a * b] * c * a * b * a } & =a *[b * a * c * a * b] * a=a *(c * a * b) * a \\
& =a *(c * a * b)=[a * b * a] * c * a * b=a * b * c * a * b
\end{aligned}
$$

as desired.

(2) We have

$$
\begin{aligned}
{[a * b] * c * a * c * a } & =a * b *[a * c * a * c * a]=a * b *[(a * c * a)] \\
& =a * b *[(a * c)]=a * b * c * a * c
\end{aligned}
$$

(3) Similar to (2).

(4) Similar to (1). 
(5) We have to check that $a * b * c * a * b * c * a=a * c * b * a * c * b$ or equivalently that $a=a * c * b * a * c * b * a * c * b * a * c * b$. Indeed,

$$
\begin{aligned}
& {[a * c] * b * a * c * b * a * c * b * a * c * b=a *[c * a * b * a * c] * b * a * c * b * a * c * b} \\
& \quad=[a *(b * a * c)] * b * a * c * b * a * c * b \\
& \quad=a *[(b * a * c)] * a * b * a * c * b * a * c * b \\
& \quad=a *[c * a * b * a * c * a * b * a * c] * b * a * c * b \\
& \quad=a *[(c *(b * a) * c)] * b * a * c * b=a *[(c *(b * a))] * b * a * c * b \\
& \quad=a * a * b * a * c * a *[* b * a * b * a] * c * b \\
& \quad=[a * a] * b * a * c *[a * a] * b * a * b * c * b=[a * b * a] * c * b * a * b * c * b \\
& \quad=a *[b * c * b * a * b * c * b]=a *(a *(c * b))=a .
\end{aligned}
$$

(6) We prove that $a * b * c * b * c * a * b * a=a * b * c * b * c * a * b$. Indeed,

$$
\begin{aligned}
a * b * c * b * c * a * b * a & =a * b * c * b * c * b *[b * a * b * a] \\
& =a *[b * c * b * c * b] * a * b * a * b \\
& =[a * c * b * c * a] * b * a * b \\
& =a *[c * b * c * b] * a * b=a * b * c * b * c * a * b .
\end{aligned}
$$

(7) $a *[b * c * b * c] * a * c * a=a * c * b * c * b * a * c * a$ and then as in (6) (the roles of $b$ and $c$ are exchanged).

(8) We need $a * b * c * a * b * c * b=a * b * c * a * b * c * b * a$ or $a * b * c * a * b * c * b * a * b * c * b * a * c * b=a$. We compute as follows:

$$
\begin{array}{rl}
{[a * b] * c} & * a * b * c * b * a * b * c * b * a * c * b \\
& =a *[b * a * c * a * b] * c * b * a * b * c * b * a * c * b \\
& =[a *(c * a * b)] * c * b * a * b * c * b * a * c * b \\
& =a *[(c * a * b)] * a * c * b * a * b * c * b * a * c * b \\
& =a * b * a * c * a * b * a * c * b * a * b * c * b * a * c * b \\
& =a * b * a * c * a * b * a * c * a *[a * b * a * b] * c * b * a * c * b \\
& =a *[b * a * c * a * b * a * c * a * b] * a * b * a * c * b * a * c * b \\
& =a *[(b *(c * a) * b)] * a * b * a * c * b * a * c * b \\
& =a *[(b * a * c * a)] * a * b * a * c * b * a * c * b \\
& =[a * a] * c * a * b * a * c *[a * a] * b * a * c * b * a * c * b \\
& =[a * c * a] * b * a * c * b * a * c * b * a * c * b \\
& =a * c * b * a * c * b * a * c * b * a * c * b \stackrel{(5)}{=} a .
\end{array}
$$


(9) $a * b * c * a * c * b * c=a * b * c * a * c * b * c * a$ is equivalent to $a * b * c * a * c * b * c * a * c * b * c * a * c * b=a$, which is proved as follows:

$$
\begin{aligned}
& {[a * b * c * a * c * b] * c * a * c * b * c * a * c * b } \\
&=a * b * c * a * c * b *[a * c * a * c] * b * c * a * c * b \\
&=a * b * c * a * c * b * c * a * c * a * b * c * a * c * b \\
&=a *[b * c * a * c * b * c * a * c * b] * b * a * b * c * a * c * b \\
&=a *[(b *(a * c) * b)] * b * a * b * c * a * c * b \\
&=[a * c * a] * c * b * c * a * c * b * a * b * c * a * c * b \\
&=a *[c * c] * b * c * a * c * b * a * b * c * a * c * b \\
&=a *[b * c * a * c * b * a * b * c * a * c * b] \\
&=a *(a * b * c * a * c * b)=a *(a *(a * c * b))=a .
\end{aligned}
$$

(10) As in (9) with $b$ and $c$ interchanged.

(11) As in (8) with $b$ and $c$ interchanged.

(12) $a * b * c * a * b * c * b * c * a=a * b * c * b * c * a * b * c$ is equivalent to $a * b * c * b * c * a * b * c * a * c * b * c * b * a * c * b=a$, which we prove as follows: $[a * b] * c * b * c * a * b * c * a * c * b * c * b * a * c * b$

$$
\begin{aligned}
= & a *[b * a * c * b * c * a * b] * c * a * c * b * c * b * a * c * b \\
= & {[a *(b * c * a * b)] * c * a * c * b * c * b * a * c * b } \\
= & a *[(b * c * a * b)] * a * c * a * c * b * c * b * a * c * b \\
= & a * b * a * c * b * c * a * b * a * c * a *[c * b * c * b] * a * c * b \\
= & {[a * b * a] * c * b * c * a * b * a * c * a * b * c * b * c * a * c * b } \\
= & {[a * b * c * b] * c * a * b * a * c * a * b * c * b * c * a * c * b } \\
= & a * b * c *[b * a * c * a * b * a * c * a * b] * c * b * c * a * c * b \\
= & a * b * c *[(b *(c * a) * b)] * c * b * c * a * c * b a * b * c * a * c * a * b \\
& * a * c * a * c * b * c * a * c * b \\
= & a * b * c * a * c * a * b * a * b *[b * c * a * c * b * c * a * c * b] \\
= & {[a * b] * c * a * c * a * b * a * b * c * a * c * b * c * a * c } \\
= & a * b *[a * c * a * c * a] * b * a * b * c * a * c * b * c * a * c \\
= & a *[b * c * a * c * b * a * b * c * a * c * b] * c * a * c \\
= & a *(a *(a * c * b)) c * a * c=a * c * a * c=a .
\end{aligned}
$$

(13) Follows from (12). 
(14) Instead of $a * b * c * b * c * a * b * c * b * a=a * b * c * b * c * a * b * c * b$ we consider $a=a * b * c * b * c * a * b * c * b * a * b * c * b * a * c * b * c * b$, proved as follows:

$$
\begin{gathered}
a * b * c * b * c *[a * b * c * b * a * b * c * b * a] * c * b * c * b \\
=a *[b * c * b * c * b * c * b * a * b * c * b * c * b * c * b] \\
=a *(a *(c *(c * b)))=a .
\end{gathered}
$$

(15) Follows from (14), since

$$
a *[b * c * b * c] * a * c * b * c * a=a * c * b * c * b * a * c * b * c * a .
$$

(16) Since $a * b * c * a * b * c * a \stackrel{(5)}{=} a * c * b * a * c * b$, we have

$$
\begin{aligned}
a * b *[c * b * c] * a * b *[c * b * c] * a & =a * b *(b * c) * a * b *(b * c) * a \\
& =a *[(b * c)] * b * a *[(b * c)] * b \\
& =a *[c * b * c * b] * a *[c * b * c * b] \\
& =a * b * c * b * c * a * b * c * b * c .
\end{aligned}
$$

The oldest open problem concerning $n$-moves is the Nakanishi 4-move conjecture (see [Prz]).

ConjeCture 9 (Nakanishi, 1979). Every knot is 4-move equivalent to the trivial knot.

Our hope was that the fourth Burnside kei of a knot could be used to detect a potential counterexample to this conjecture. However, the following theorem suggests this is not likely to be the case.

THEOREM 10. Every algebraically connected quotient of $\bar{Q}(3,4)$ is a trivial quandle (a quandle with one element).

Proof. Let $\widetilde{Q}$ be the algebraically connected quotient of $\bar{Q}(3,4), f: \bar{Q}(3,4)$ $\rightarrow \widetilde{Q}$ be the quotient homomorphism, and $S_{1}, S_{2}, S_{3}$ denote the algebraically connected components of $\bar{Q}(3,4)$. We claim that $\widetilde{Q}$ is contained in each image $f\left(S_{i}\right)$ for $i=1,2,3$.

Assume that there exists $x \in \widetilde{Q}$ with $f^{-1}(x) \cap S_{j}=\emptyset$ for some $j$. Let $a \in S_{j}$ and $y=f(a)$. Then from the algebraic connectivity of $\widetilde{Q}$ it follows that $x=y * x_{1} * \cdots * x_{k}$ for some $x_{1}, \ldots, x_{k} \in \widetilde{Q}$. Now we choose arbitrary $z_{i} \in f^{-1}\left(x_{i}\right), i=1, \ldots, k$. Let $z=a * z_{1} * \cdots * z_{k}$. Then $z \in S_{j}$ and $f(z)=f(a) * f\left(z_{1}\right) * \cdots * f\left(z_{k}\right)=y * x_{1} * \cdots * x_{k}=x$, which contradicts the assumption that $f^{-1}(x) \cap S_{j}=\emptyset$.

Each subquandle $S_{i}$, when considered as a quandle itself, has eight 4element components (orbits) $T_{1}, \ldots, T_{8}$ and, just as before, we can prove that $\widetilde{Q}$ is the image of each $T_{i}$ (and can have at most 4 elements). But every 
such $T_{i}$ is a trivial quandle $\left(x * y=x\right.$ for any $\left.x, y \in T_{i}\right)$ and $\widetilde{Q}$ must be the image of just one element.

Since knot quandles are algebraically connected, we have the following result.

Corollary 11. Let $K$ be a knot such that the minimal number of generators of its fundamental quandle is less than or equal to 3 . Then its fourth Burnside quandle, $\bar{Q}_{4}(K)$, has only one element.

For example, $\bar{Q}_{4}(K)$ will not detect a potential counterexample to the Nakanishi 4-move conjecture among 3-bridge knots.

It seems plausible that the order of $\bar{Q}_{4}(K)$ is 1 for any knot $K$.

Acknowledgements. We would like to thank Tomotada Ohtsuki for several very useful discussions in December 2004 and January 2005, especially for informing us about his calculations regarding $\bar{Q}(4,3)$. We would also like to thank Michael Kinyon for a very informative email [Kin]. In particular we thank him for pointing out to us that the relations $r_{n}$ have a long history in the theory of quasigroups (see [Sta] for background information). We are indebted to Seiichi Kamada for translating for us parts of Takasaki's paper. We wish to thank the referee for the report that helped to improve our paper.

\section{References}

[Bel] V. D. Belousov, Foundations of the Theory of Quasigroups and Loops, Nauka, Moscow, 1967 (in Russian).

[Bu] W. Burnside, On an unsettled question in the theory of discontinuous groups, Quart. J. Pure Appl. Math. 33 (1902), 230-238.

[C-E-S] J. S. Carter, M. Elhamdadi and M. Saito, Twisted quandle homology theory and cocycle knot invariants, Algebr. Geom. Topol. 2 (2002), 95-135.

[Dab] M. K. Dąbkowski, e-mail communication, November 25-26th, 2004.

[D-I-P] M. K. Dąbkowski, M. Ishiwata and J. H. Przytycki, Rational moves and tangle embeddings: (2,2)-moves as a case study, in: Proc. Conf. Topology of Knots VII (held at TWCU, December 23-26), February, 2005, 37-46 (in Japanese); e-print (in English): http://front.math.ucdavis.edu/math.GT/0501539.

[F-R] R. Fenn and C. Rourke, Racks and links in codimension two, J. Knot Theory Ramif. 1 (1992), 343-406.

[GAP4] The GAP Group, GAP-Groups, Algorithms, and Programming, Version 4.4, 2005 (http://www.gap-system.org).

[Joy] D. Joyce, A classifying invariant of knots: the knot quandle, J. Pure Appl. Algebra 23 (1982), 37-65.

[Kam] S. Kamada, Knot invariants derived from quandles and racks, Geom. Topol. Monogr. 4 (2002), 103-117.

[Kin] M. Kinyon, e-mail communication, January 3, 2006.

[Oht] T. Ohtsuki, personal communication, December 2004 and January 2005. 
[Prz] J. H. Przytycki, $t_{k}$-moves on links, in: Braids, J. S. Birman and A. Libgober (eds.), Contemp. Math. 78, Amer. Math. Soc., 1988, 615-656.

[Sta] D. Stanovský, Left distributive left quasigroups, $\mathrm{PhD}$ thesis, Charles Univ. Prague, 2004.

[Tak] M. Takasaki, Abstraction of symmetric transformation, Tôhoku Math. J. 49 (1942/3), 145-207 (in Japanese).

[VL] M. Vaughan-Lee, The Restricted Burnside Problem, 2nd ed., London Math. Soc. Monogr. 8, Clarendon Press, Oxford Univ. Press, New York, 1993.

Department of Mathematics

The George Washington University

Old Main Bldg, 1922 F St. NW

Washington, DC 20052, U.S.A.

E-mail: niebrz@gwu.edu

przytyck@gwu.edu

Received 30 December 2005;

in revised form 30 January 2006 